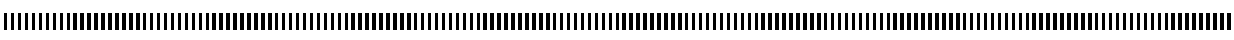

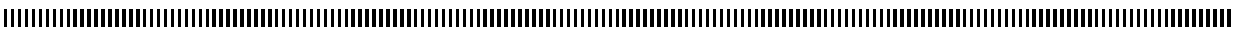

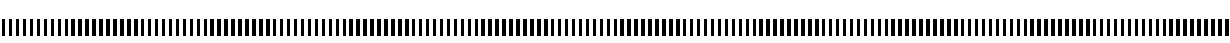

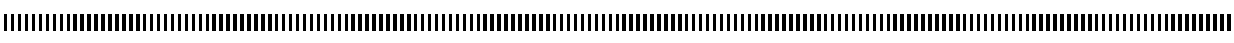

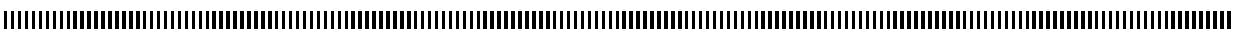

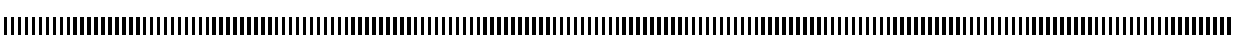

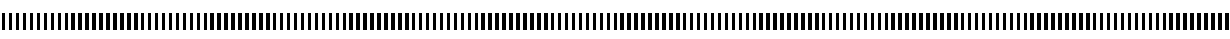

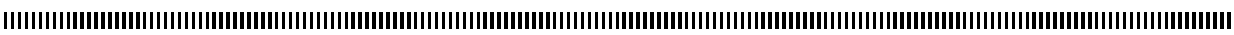

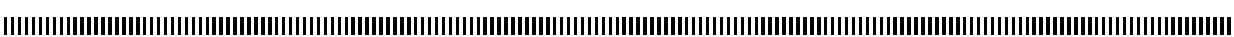

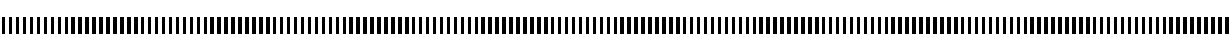

Special issue CARI'12

\title{
Vers une structuration auto-stabilisante des réseaux Ad Hoc
}

\author{
Mandicou Ba* - Olivier Flauzac* — Bachar Salim Haggar* \\ Rafik Makhloufi $\ddagger$ - Florent Nolot* — Ibrahima Niang $\dagger$ \\ * Université de Reims Champagne-Ardenne \\ Laboratoire CReSTIC - Équipe SysCom EA 3804 \\ \{mandicou.ba, olivier.flauzac, bachar-salim.haggar, florent.nolot\}@univ-reims.fr \\ $\dagger$ Université Cheikh Anta Diop \\ Département de Mathématiques et Informatique \\ ‡École Nationale des Ponts et Chaussées \\ Laboratoire CERMICS \\ Laboratoire d'Informatique de Dakar (LID) \\ iniang@ucad.sn \\ Laboratoire CERMICS - Equipe SOWG \\ makhlour@cermics.enpc.fr
}

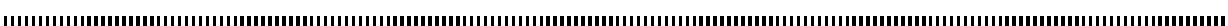

RÉSUMÉ. Dans cet article, nous proposons un algorithme de structuration auto-stabilisant, distribué et asynchrone qui construit des clusters de diamètre au plus $2 k$. Notre approche ne nécessite aucune initialisation. Elle se fonde uniquement sur l'information provenant des nœuds voisins à l'aide d'échanges de messages. Partant d'une configuration quelconque, le réseau converge vers un état stable après un nombre fini d'étapes. Nous montrons par preuve formelle que pour un réseau de $n$ nœuds, la stabilisation est atteinte en au plus $n+2$ transitions. De plus, l'algorithme nécessite une occupation mémoire de $\left(\Delta_{u}+1\right) * \log (2 n+k+3)$ bits pour chaque nœud $u$ où $\Delta_{u}$ représente le degré (nombre de voisins) de $u$ et $k$ la distance maximale dans les clusters. Afin de consolider les résultats théoriques obtenus, nous avons effectué une campagne de simulation sous $\mathrm{OMNeT++}$ pour évaluer la performance de notre solution.

ABSTRACT. In this paper, we present a self-stabilizing asynchronous distributed clustering algorithm that builds non-overlapping k-hops clusters. Our approach does not require any initialization. It is based only on information from neighboring nodes with periodic messages exchange. Starting from an arbitrary configuration, the network converges to a stable state after a finite number of steps. Firstly, we prove that the stabilization is reached after at most $n+2$ transitions and requires $\left(\Delta_{u}+1\right) *$ $\log (2 n+k+3)$ bits per node, where $\Delta_{u}$ represents node's degree, $n$ is the number of network nodes and $k$ represents the maximum hops number. Secondly, using OMNet++ simulator, we performed an evaluation of our proposed algorithm.

MOTS-CLÉS : Réseaux Ad Hoc, clustering, algorithmes distribués, auto-stabilisation, OMNeT++.

KEYWORDS : Ad hoc networks, clustering, distributed algorithms, self-stabilizing, OMNeT++. 


\section{Introduction}

Dans les réseaux Ad Hoc, la solution de communication la plus utilisée est la diffusion. C'est une technique simple qui nécessite peu de calcul. Cependant, elle est coûteuse en termes d'échanges de messages et peut entraîner une saturation du réseau. Afin d'optimiser les communications, une solution efficace consiste en la structuration du réseau en arbres [9] ou en clusters [15].

Plusieurs travaux comme [20, 32, 33] ont montré que le clustering offre une structuration efficace du réseau permettant ainsi d'optimiser les communications. Le clustering consiste à découper le réseau en groupes de nœuds appelés clusters donnant ainsi au réseau une structure hiérarchique [22]. Chaque cluster est représenté par un nœud particulier appelé cluster-head. Un nœud est élu cluster-head selon une métrique telle que le degré, la mobilité, l'identité des nœuds, la densité, etc. ou une combinaison de ces paramètres.

En outre, les réseaux Ad Hoc peuvent être également dynamiques. Un nœud peut se connecter ou se déconnecter du réseau à tout instant et peut donc induire des fautes transitoires par modification de la topologie du réseau. Donc, concevoir des solutions de clustering tolérantes aux fautes transitoires est plus que nécessaire pour des applications de réseaux Ad Hoc souvent critiques. Dans [21], Johnen et al. ont montré que l'autostabilisation, introduite par Dijkstra en 1974 dans [17], est une propriété intéressante de tolérance aux fautes transitoires dans un système distribué comme les réseaux Ad Hoc. Dijkstra considère qu'un système est auto-stabilisant si quelle que soit sa configuration de départ, il est garanti d'arriver à une configuration légale en un nombre fini d'étapes. Suite à l'apparition d'une faute transitoire, le système de lui même, retrouve une configuration légale après un temps fini. Donc, les algorithmes de clustering auto-stabilisants sont une solution efficace pour structurer le réseau dans le but d'optimiser les communications et de résister aux fautes transitoires qui peuvent survenir.

Plusieurs solutions de clustering auto-stabilisantes ont été proposées dans la littérature $[13,16,19,23,24,25,26,27,28]$. Elles sont classées en algorithmes à 1 saut ou à $k$ sauts. Dans les solutions à 1 saut $[19,23,24,25,28]$, les nœuds sont à distance 1 du cluster-head et le diamètre maximal des clusters est de 2. Par contre, dans les solutions à $k$ sauts $[13,16,27,26]$, les nœuds peuvent se situer jusqu'à une distance $k$ du cluster-head et le diamètre maximal des clusters est donc au plus de $2 k$. En outre, les solutions existantes utilisent soit un modèle à mémoire partagée (modèle à états ou modèle à registres) $[13,16,23,24]$ soit un modèle à passage de messages [13, 16, 26, 27]. Les solutions qui utilisent un modèle à états, comme elles ne se focalisent pas sur les communications (envoi et réception de messages), elles ne sont donc pas réalistes dans le cadre des réseaux AdHoc. Pour les solutions fondées sur un modèle à passage de messages, bien qu'étant plus réalistes dans le contexte des réseau Ad Hoc, elles sont souvent coûteuses en termes d'échanges de messages. Or, plusieurs études, comme Pottie et Kaiser dans [29], ont montré que les communications représentent la principale source de consommation de ressources énergétiques.

Ainsi, dans le but de réduire les communications et de résister aux fautes transitoires, nous proposons, dans cet article, un algorithme de clustering à $k$ sauts qui est complètement distribué et auto-stabilisant. Notre solution utilise un modèle asynchrone à passage de messages et construit des clusters non-recouvrants de diamètre au plus $2 k$. Elle ne nécessite pas d'initialisation et est tolérante aux fautes transitoires. De plus, nous utilisons le 
critère de l'identité maximale des nœuds pour l'élection du cluster-head qui apporte une meilleure de stabilité. Pour les communications, nous considérons seulement un échange de messages avec le voisinage à distance à distance 1 (vue locale) pour construire des clusters à $k$ sauts.

La suite de l'article est organisée comme suit. Dans la Section 2, nous étudions les solutions de clustering existantes. La Section 3 présente notre contribution. A la Section 4, nous décrivons le modèle sur lequel se fonde notre approche. Puis, à la section 5 , nous donnons le principe d'exécution et les détails de notre algorithme. Dans la Section 6, nous montrons le schéma de la preuve de convergence, de clôture et de l'occupation mémoire de notre algorithme. Nous présentons une campagne de simulation sous OMNeT++ dans la Section 7 pour évaluer les performances moyennes de notre solution. Une conclusion et des perspectives sont données dans la Section 8 .

\section{2. État de l'art}

Plusieurs propositions de clustering ont été faites dans la littérature [13, 16, 19, 23, $24,25,26,27,28]$.

Les approches auto-stabilisantes [25, 19, 24, 23, 28] construisent des clusters à 1 saut. Mitton et al. [28] utilisent comme métrique la densité, dans un modèle asynchrone à passage de messages, afin de minimiser la reconstruction de la structure en cas de faible changement de topologie. Chaque nœud calcule sa densité et la diffuse à ses voisins situés à distance 1 . Le nœud avec la plus grande densité dans son voisinage devient clusterhead. Dans cette approche, un nœud avec une forte mobilité mobile ne s'attache à aucun cluster. De plus, du fait du calcul et de la diffusion de la densité, cette approche engendre d'importantes communications. Flauzac et al. ont proposé dans [19] un algorithme autostabilisant, dans un modèle asynchrone à passage de messages, qui combine la découverte de topologie et de clustering en une seule phase. Elle ne nécessite qu'un seul type de message échangé entre voisins. Un nœud devient cluster-head s'il possède la plus grande identité parmi tous ses voisins.

Dans [10, 11], Bui et al. ont proposé un algorithme de clustering adaptatif aux changements de topologie mais non auto-stabilisant et non déterministe. Ils utilisent une marche aléatoire pour construire d'abord un cœur de cluster composé de 2 à MaxCoreSize nœuds. Ce cœur est ensuite étendu aux voisins immédiats, appelés nœuds ordinaires, pour former les clusters. Les approches de Bui et al. [10,11], comme elles utilisent la marche aléatoire les communications, sont coûteuses en termes de messages échangés.

Johnen et al. ont proposé dans [24] un algorithme auto-stabilisant qui se fonde sur un modèle à états, qui construit des clusters de taille fixe. Les auteurs attribuent un poids à chaque nœud et fixent un paramètre SizeBound qui représente le nombre maximal de nœuds dans un cluster. Un nœud ayant le poids le plus élevé devient cluster-head et collecte dans son cluster jusqu'à SizeBound nœuds. Dans [23], Johnen et al. ont étendu leur proposition décrite dans [24] pour apporter la notion de robustesse. La robustesse est une propriété qui assure qu'en partant d'une configuration quelconque, le réseau est partitionné après un round asynchrone. Durant la phase de convergence, le réseau demeure toujours partitionné et vérifie un prédicat de sûreté. Dans [25], Kuroiwa et al. améliorent l'approche proposée par Johnen et al. dans [24]. Kuroiwa et al. proposent une méthode fondée sur un modèle à états synchrone afin d'apporter une meilleure stabilité dans la 
structure des clusters en comparaison avec la solution décrite [24]. Les solutions proposées dans [24, 24, 25], utilisent un modèle à états, ne sont pas réalistes dans le cadre des réseaux Ad Hoc.

Les approches auto-stabilisantes [27, 26, 13, 16] construisent des clusters à $k$ sauts.

Dans [27], Miton et al. ont étendu leurs travaux décrits dans [28] pour proposer un algorithme robuste de clustering auto-stabilisant à $k$ sauts. Miton et al. utilisent un modèle asynchrone à passage de messages. Chaque nœud du réseau calcule sa $k$-density en utilisant les information de son voisinage à distance $k+1$ sauts $(\{k+1\}$-Neighborhood view). Puis, la $k$-density de chaque nœud est retransmise à distance $k$. Le nœuds avec la plus grande valeur de $k$-density devient cluster-head. Dans cet algorithme, un nœud trop mobile ne s'attache à aucun cluster. De plus, du fait du calcul et de la diffusion de la $k$-density jusqu'à une distance $k$, cette solution est coûteuse en termes d'échanges de messages.

Larsson et Tsigas ont proposé dans [26] un algorithme distribué, auto-stabilisant avec multiple chemins nommé $(x, k)$-clustering. Cet algorithme assigne, si possible, $x$ clusterheads dans une distance de $k$ sauts à tout nœud du réseau. Les auteurs utilisent un modèle synchrone à passage de messages et procèdent par rounds. Ils ont montré que leur algorithme attribue, si possible, $x$ cluster-heads à chaque nœud en $O(k)$ rounds. Puis, l'ensemble des cluster-heads se stabilise, avec une forte probabilité, en un minimum local de $O(g * k * \log (n))$ rounds. De plus, il nécessite $O\left(\left|G_{u}^{k}\right| *(\log (n)+\log (k))\right)$ bits pour chaque nœuds $u$ du réseau, où $n$ représente la taille du réseau, $k$ le nombre de maximal de sauts et $g$ une borne supérieure du nombre de nœuds dans un cluster. Cette approche génère beaucoup de messages. En effet, les messages de chaque nœuds sont retransmis jusqu'à une distance $k$.

Caron et al. [13], utilisant comme métrique un unique identifiant pour chaque nœud avec un poids attribué à chaque arrête du graphe, ont proposé un algorithme nommé $k$ clustering et fondé sur un modèle à états. Un $k$-clustering dans un graphe consiste à partitionner le réseau en clusters disjoints dans lesquels chaque nœud se situe au plus à distance $k$ du cluster-head. Cette solution s'inspire partiellement dans l'algorithme de Amis et al. proposé dans [3]. Dans cette solution chaque nœud peut lire les états de ses voisins situés jusqu'à une distance $k+1$ et est le seul à disposer des privilèges d'écriture sur ses propres états. Les auteurs ont montré que cet algorithme s'exécute en $O(n * k)$ rounds et nécessite $O(\log (n)+\log (k))$ bits par nœud, où $n$ est le nombre de nœuds du réseau.

Dans [16], utilisant le critère de l'identité minimale, Datta et al. ont proposé un algorithme, nommé MINIMAL, de clustering auto-stabilisant à $k$ sauts. MINIMAL utilise un modèle à états et considère un graphe $G$ quelconque de $n$ nœud avec un identifiant unique attribué à chacun. MINIMAL construit d'abord un ensemble $D$ qui représente un $k$-dominating. $D$ est défini tel que tout nœud n'appartenant pas à $D$ se situe au plus à distance $k$ d'au moins d'un membre de $D$. Datta et $a l$. ont prouvé que, pour construire $D$, MINIMAL requiert $O(n)$ rounds. Puis, considérant $D$ comme l'ensemble de clusterheads, MINIMAL structure $G$ en clusters de rayon $k$ et ceci nécessite $O\left(n^{2}\right)$ rounds. De plus, il requiert $\log (n)$ bits pour tout nœud de $G$. 


\section{Contribution}

Les solutions de clustering auto-stabilisantes à $k$ qui se fondent sur un modèle à états [13, 16, 24, 23] nécessitent un temps de stabilisant élevé. De plus, comme elles ne se focalisent pas sur les envois et réceptions de messages, elles ne sont pas réalistes dans le cadre des réseaux $A d H o c$. Quant aux solutions qui utilisent un modèle à passage de messages [25, 24, 23, 28], bien qu'elles sont plus réalistes dans le contexte des réseaux $A d$ $H o c$, elles sont couteuses en termes d'échanges de messages. Or, dans les réseaux Ad Hoc, les communications représentent la majeure source de consommation de ressources [29].

Ainsi, nous proposons une approche de structuration qui utilise un modèle asynchrone à passage de messages contrairement aux solutions proposées dans [24, 23, 13, 16, 25] . Notre solution est distribuée et auto-stabilisante. Nous utilisons le critère d'identité maximale qui apporte plus de stabilité par rapport aux métriques variables utilisées dans [27, $28,24,23]$. Notre algorithme structure le réseau en clusters disjoints deux à deux et de diamètre au plus égal à $2 k$. Cette structuration ne nécessite aucune initialisation. Nous combinons la découverte de voisinage et le clustering en une seule phase. Nous utilisons uniquement les informations provenant des nœuds voisins situés à distance 1 , par le biais d'échanges de messages, pour construire des clusters à $k$ sauts.

D’une part, par une preuve formelle, nous montrons que pour un réseau de $n$ nœuds, la stabilisation est atteinte dans le pire des cas en $n+2$ transitions. De plus, il nécessite une occupation mémoire de $\left(\Delta_{u}+1\right) * \log (2 n+k+3)$ bits pour chaque nœud $u$ où $\Delta_{u}$ représente le degré - nombre de voisins - de $u, n$ le nombre total de nœuds et $k$ la distance maximale dans les clusters. D'autre part, par une campagne de simulation sous $\mathrm{OMNeT}++$, nous évaluons les performances moyennes de notre solution. Le résultats de simulation montrent qu'en moyenne, nous obtenons des temps de stabilisation très inférieurs à celui du pire cas. De plus, suite à l'occurrence de fautes transitoires, le coût de la restructuration et le nombre de nœuds impliqués dans cette opération sont moindres.

\section{Modélisation}

Nous considérons notre réseau comme un système distribué que nous modélisons par un graphe connexe et non orienté $G=(V, E)$ [4, 30]. $V$ désigne l'ensemble des nœuds du réseau avec $|V|=n$ et $n \geq 2$. $E$ représente l'ensemble des connexions existantes entre les nœuds. Nous supposons que chaque nœud $u \in V$ est caractérisé par une «valeur d'identification » qui lui est propre; son identité que nous notons $i d_{u}$ et telle que $0 \leq i d_{u} \leq n-1$. Cette identité est tirée d'un ensemble $\mathcal{I}$ non vide fini ou infini mais obligatoirement muni d'un ordre total qui peut être, par exemple, l'ordre numérique sur $\mathbb{N}$. Soient $u$ et $v$ deux nœuds appartenant à $V$. Une arête $(u, v)$ existe si et seulement si $u$ peut communiquer avec $v$ et vice-versa. Ce qui implique que tous les liens sont bidirectionnels. Dans ce cas, les nœuds $u$ et $v$ sont voisins. L'ensemble des nœuds $v \in V$ voisins du nœud $u$ situés à distance 1 est noté $N_{u}$. On définit la distance $d_{(u, v)}$ entre deux nœuds $u$ et $v$ quelconque dans le graphe $G$ comme le nombre d'arêtes minimal le long du chemin entre $u$ et $v$.

Nous utilisons un modèle de communication asynchrone à passage de messages. Ce choix est motivé par la validité de ce modèle; validité due à sa similarité réalité (les réseaux $\mathrm{Ad} H o c$ ) comparé au modèle à états. Ainsi, chaque paire de nœuds $(u, v)$ du réseau 
est connectée par un canal de communication bidirectionnel. Les canaux de communication sont asynchrones; le temps de transite des messages est fini et non borné. De plus, nous considérons ces canaux de communication comme fiables; pas de perte ni de corruption de messages. Chaque nœud $u$ envoie périodiquement à ses voisins $v \in N_{u}$ un message contenant les informations sur son état actuel. Nous supposons qu'un message envoyé est correctement reçu au bout d'un temps fini mais non borné par tous les voisins situés à distance 1 . De plus, chaque nœud $u$ du réseau maintient dans une table de voisinage les états actuels de ses voisins. La réception d'au moins d'un message d'un voisin $v$ déclenche l'exécution de notre algorithme de clustering.

\section{Structuration auto-stabilisante dans les réseaux Ad Hoc}

\subsection{Préliminaires}

Dans cette section, nous donnons les différents concepts et définitions utilisés dans notre approche.

\section{Définition 5.1 (Cluster)}

Nous définissons un cluster à $k$ sauts comme un sous graphe connexe du réseau, dont le diamètre est inférieur ou égal à $2 k$. L'ensemble des nœuds d'un cluster u est noté $V_{u}$.

\section{Définition 5.2 (Identifiant du cluster)}

Chaque cluster possède un unique identifiant correspondant à la plus grande identité de tous les nœuds du cluster. L'identité d'un cluster auquel appartient le nœud u est notée $c l_{u}$.

Dans nos clusters (cf. Figure 1(a)), chaque nœud $u$ possède un statut noté statut (cf. $_{\text {. }}$ Figure 1(b)) qui définit son rôle à jouer. Ainsi, un nœud peut être soit Cluster-Head $(\mathrm{CH})$, soit Simple Node $(S N)$, ou soit Gateway Node $(G N)$. Le cluster-head est le nœud possédant la plus grande identité dans le cluster. Il a pour rôle de manager les communications au sein du cluster. Les nœuds gateway nodes assurent l'interconnexion entre clusters voisins et permettent les communications extra-clusters. Un nœud qui n'est ni cluster-head ni gateway node est alors qualifié de simple node d'un cluster. De plus, chaque nœud $u$ adopte un voisin $v \in N_{u}$, noté $g n_{u}$, par lequel il passe pour atteindre son $C H$.

Définition 5.3 (Statut des nouds)

- Cluster-Head $(\boldsymbol{C H})$ : un noud u a le statut de CH s'il possède le plus grand identifiant parmi tous les nouds de son cluster:

- statut $_{u}=C H \Longleftrightarrow \forall v \in V_{c l_{u}},\left(i d_{u}>i d_{v}\right) \wedge\left(\operatorname{dist}_{(u, v)} \leq k\right)$.

- Simple Node (SN) : un noud u a le statut de $S N$ si tous ses voisins appartiennent au même cluster que lui :

- statut $_{u}=S N \Longleftrightarrow\left(\forall v \in N_{u}, c l_{v}=c l_{u}\right) \wedge\left(\exists w \in V /\left(\right.\right.$ statut $\left._{w}=C H\right) \wedge$ $\left.\left(\operatorname{dist}_{(u, w)} \leq k\right)\right)$.

- Gateway Node (GN) : un nœud u a le statut de GN s'il existe un nœud $v$ dans son voisinage appartenant un autre cluster :

- statut $_{u}=G N \Longleftrightarrow \exists v \in N_{u},\left(c_{v} \neq c_{u}\right)$. 


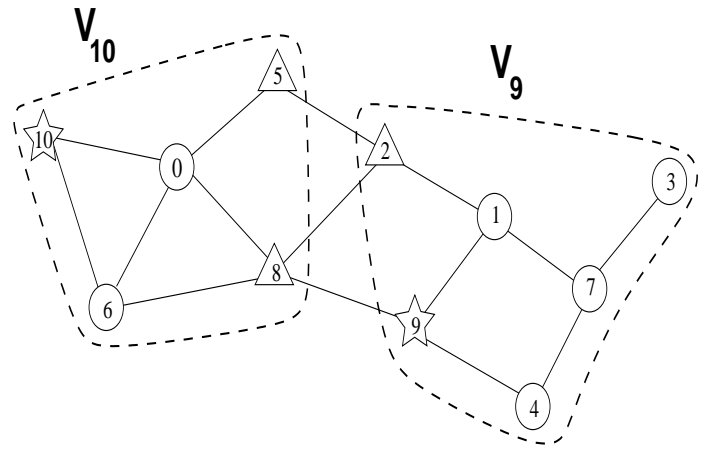

(a) Structuration en clusters

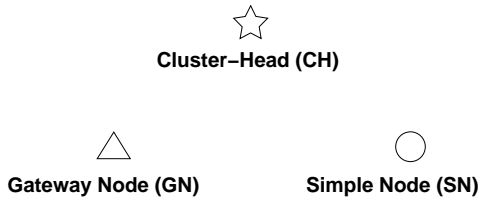

(b) Statut des nœuds

Figure 1 - Structure des clusters et statuts des nœuds

Définition 5.4 (Nouud cohérent)

Un noud u est cohérent (cf. Figure 2(b)) si et seulement si, il est dans l'un des états suivants :

- Si statut ${ }_{u}=C H$ alors $\left(c l_{u}=i d_{u}\right) \wedge\left(\operatorname{dist}_{\left(u, C H_{u}\right)}=0\right) \wedge\left(g n_{u}=i d_{u}\right)$.

- Si statut ${ }_{u} \in\{S N, G N\}$ alors $\left(c l_{u} \neq i d_{u}\right) \wedge\left(\operatorname{dist}_{\left(u, C H_{u}\right)} \neq 0\right) \wedge\left(g n_{u} \neq i d_{u}\right)$.

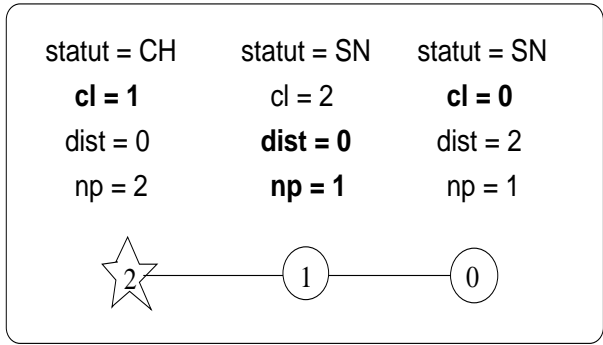

(a) Nœuds incohérents

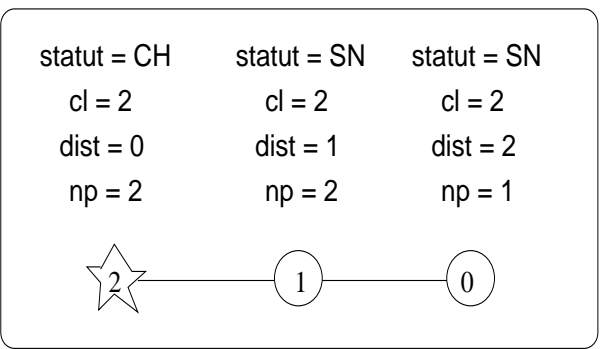

(b) Nœuds cohérents

Figure 2 - Notion de cohérence des nœuds

\section{Définition 5.5 (Noud stable)}

Un noud u est dans un état stable si et seulement si, il est cohérent et satisfait l'une des conditions suivantes:

- Si statut ${ }_{u}=C H$ alors $\left\{\forall v \in N_{u}\right.$ statut $\left._{v} \neq C H\right\} \wedge\left\{\forall v \in N_{u}\right.$ tel que cl $_{v}=$ cl $_{u}$ alors $\left.\left(i d_{v}<i d_{u}\right)\right\} \wedge\left\{\forall v \in N_{u}\right.$ tel que $i d_{v}>i d_{u} \operatorname{alors}\left(\operatorname{cl}_{v} \neq \operatorname{cl}_{u}\right) \wedge\left(\operatorname{dist}_{\left(v, C H_{v}\right)}=\right.$ $k)\}$.

- Si statut ${ }_{u}=S N$ alors $\left\{\forall v \in N_{u},\left(c_{v}=c_{u}\right) \wedge\left(\operatorname{dist}_{\left(u, C H_{u}\right)} \leq k\right)\right\} \wedge\{\exists v \in$ $\left.N_{u},\left(g n_{v}=i d_{u}\right) \wedge\left(\operatorname{dist}_{\left(v, C H_{v}\right)}=\operatorname{dist}_{\left(u, C H_{u}\right)}+1\right)\right\}$.

- Si statut $_{u}=G N$ alors $\exists v \in N_{u},\left(c l_{v} \neq c l_{u}\right) \wedge\left\{\left(\operatorname{dist}_{\left(u, C H_{u}\right)}=k\right) \vee\right.$ $\left.\left.\operatorname{dist}_{\left(v, C H_{v}\right)}=k\right)\right\}$.

\section{Définition 5.6 (Réseau stable)}

Le réseau est stable si et seulement si tous les nouds sont stables (cf. Figure 3). 


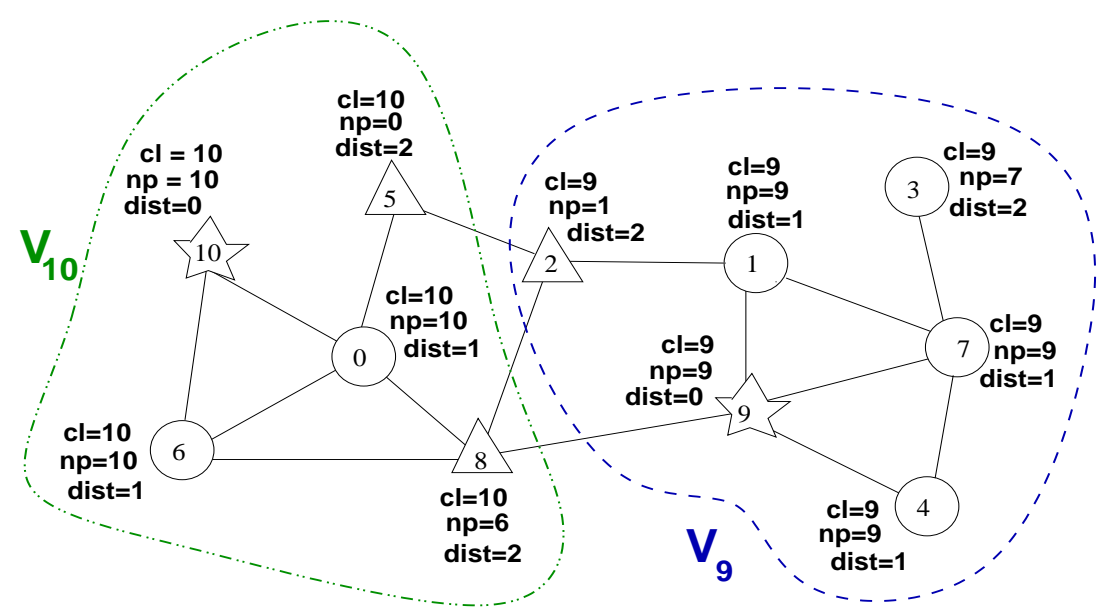

Figure 3 - Réseau stable

\subsection{Principe d'exécution}

Notre algorithme est auto-stabilisant, il ne nécessite ainsi aucune initialisation. Partant d'une configuration quelconque, avec seulement l'échange d'un seul type de message, les nœuds s'auto-organisent en clusters non-recouvrants au bout d'un nombre fini d'étapes. Ce message, dit hello, est échangé entre chaque pair de nœuds voisins. Il contient les quatre informations suivantes : l'identité du nœud $u$ expéditeur $\left(i d_{u}\right)$, son identifiant de cluster $\left(c l_{u}\right)$, son statut $\left(\right.$ statut $\left._{u}\right)$ et la distance $\left(\operatorname{dist}_{\left(u, C H_{u}\right)}\right)$ qui le sépare de son cluster-head. Rappelons que l'identifiant du cluster d'un nœud est égal à l'identifiant de son cluster-head (cf. Définition 5.2). Donc, la structure des messages hello est la suivante : hello $\left(i d_{u}, c l_{u}\right.$, statut $\left._{u}, \operatorname{dist}_{\left(u, C H_{u}\right)}\right)$. De plus, chaque nœud maintient une table de voisinage, notée StateNeigh $h_{u}$, contenant l'ensemble des états de ses voisins. StateNeigh $h_{u}[v]$ contient les états du nœud $v$ voisin du nœud $u$

La solution que nous proposons se déroule de la façon suivante :

Dès la réception d'un message hello, un nœud $u$ exécute l'algorithme 1 . Durant cet algorithme, $u$ exécute trois étapes consécutives. D'abord, il effectue la mise à jour de sa table de voisinage, ensuite il vérifie la cohérence de ses variables locales et en dernier il procède la phase de clustering. A la fin de cette exécution, $u$ envoie un message hello à touts ses voisins.

Après la phase de mise à jour, chaque nœud vérifie sa cohérence. Comme le clusterhead est le nœud avec la plus grande identité au sein du cluster, si un nœud a le statut de $\mathrm{CH}$ alors son identifiant de cluster doit obligatoirement être égal à son propre identifiant. Dans la figure 2(a), le nœud d'identité 2 est cluster-head, son identifiant de cluster est égal à 1 donc le nœud 2 n'est pas cohérent. Tout comme les nœuds 1 et 0 , ils ne vérifient pas la définition 5.4. Chaque nœud détecte et corrige son incohérence durant la phase de vérification de cohérence telle que définit dans l'algorithme 1. La figure 2(b) montre les nœuds dans des états cohérents.

Durant l'étape de clustering, chaque nœud compare son identité à celle de touts ses voisins. Un nœud $u$ s'élit cluster-head s'il la plus grand identité parmi tous les nœuds de son cluster. Si nœud $u$ découvre un voisin $v$ avec une plus grande identité que la sienne, alors il devient membre du même cluster que $v$ avec un statut de $S N$. Si un nœud reçoit 
un message provenant d'un voisin membre d'un autre cluster, alors il devient un nœud de passage avec un statut de $G N$. Comme les messages hello contiennent la distance entre chaque nœud $u$ et son cluster-head, alors $u$ peut déterminer si le diamètre maximal du cluster est atteint. Ainsi $u$ pourra donc choisir un autre cluster si les $k$ sauts sont atteints.

La figure 4 illustre le passage d'une configuration $\gamma_{i}$ à $\gamma_{i+1}$. Dans cet exemple, $k=2$. A $\gamma_{i}$, chaque nœud envoie à ses voisins un message hello. $\gamma_{i+1}$ est une configuration stable. Dès la réception de messages venant des voisins, chaque nœud met à jour sa table de voisinage puis exécute l'algorithme 1 . Dans cet exemple, le nœud $n_{1}$ qui est un nœud membre du cluster de $n_{3}$ détecte le nœud $n_{0}$ comme un cluster voisin. Il devient nœud de passage avec un statut de $G N$ et envoie un message hello à ses voisins pour une mise à jour. Les nœuds $n_{3}, n_{2}$ et $n_{0}$, en fonction de leurs états actuels ainsi que ceux de leurs voisins, ne changent pas d'état. $\gamma_{i+i}$ correspond à un état stable.

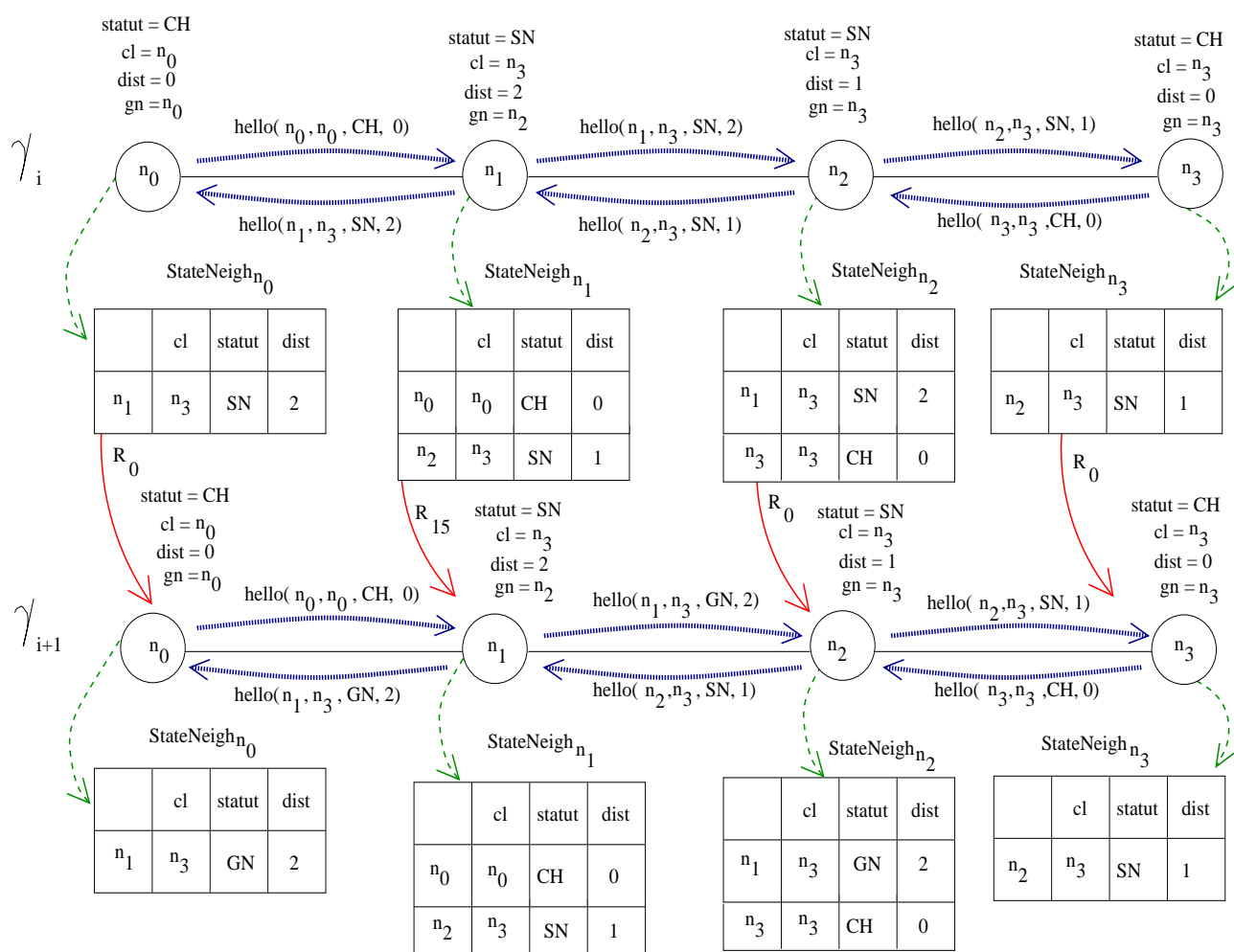

Figure 4 - Passage d'une configuration $\gamma_{i}$ à $\gamma_{i+1}$

\subsection{Algorithme auto-stabilisant de clustering à $\mathrm{k}$ sauts}

Chaque nœud $u$ du réseau connaît le paramètre $k$ avec $k<n$ qui représente le nombre maximal de saut dans un cluster. De plus, tout nœud $u$ possède les variables locales et macros qui sont définis dans le tableau 1. L'exécution de l'algorithme 1 est déclenchée par la réception d'au moins d'un message venant d'au moins d'un voisin $v$. Suite à cette exécution, chaque nœud $u$ envoie un message à ses voisins pour les informer de son changement d'état. 


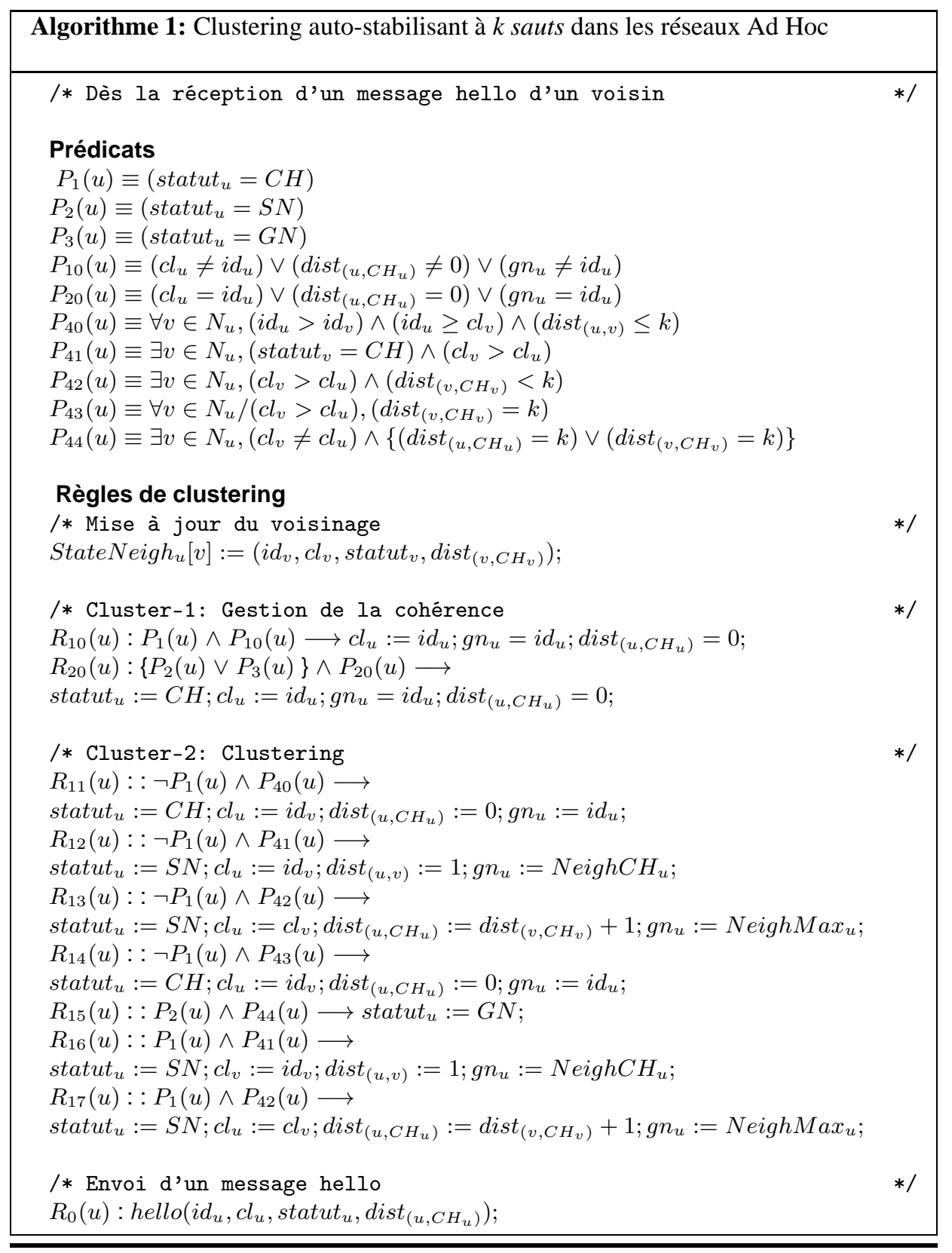

\section{Schéma intuitif de la preuve de la stabilisation}

Dans cette section, nous énonçons les principaux théorèmes et propriétés vérifiés par notre algorithme. Nous ne donnons pas tous les détails des preuves. Nous avons publié la preuve au complète dans [5]. 


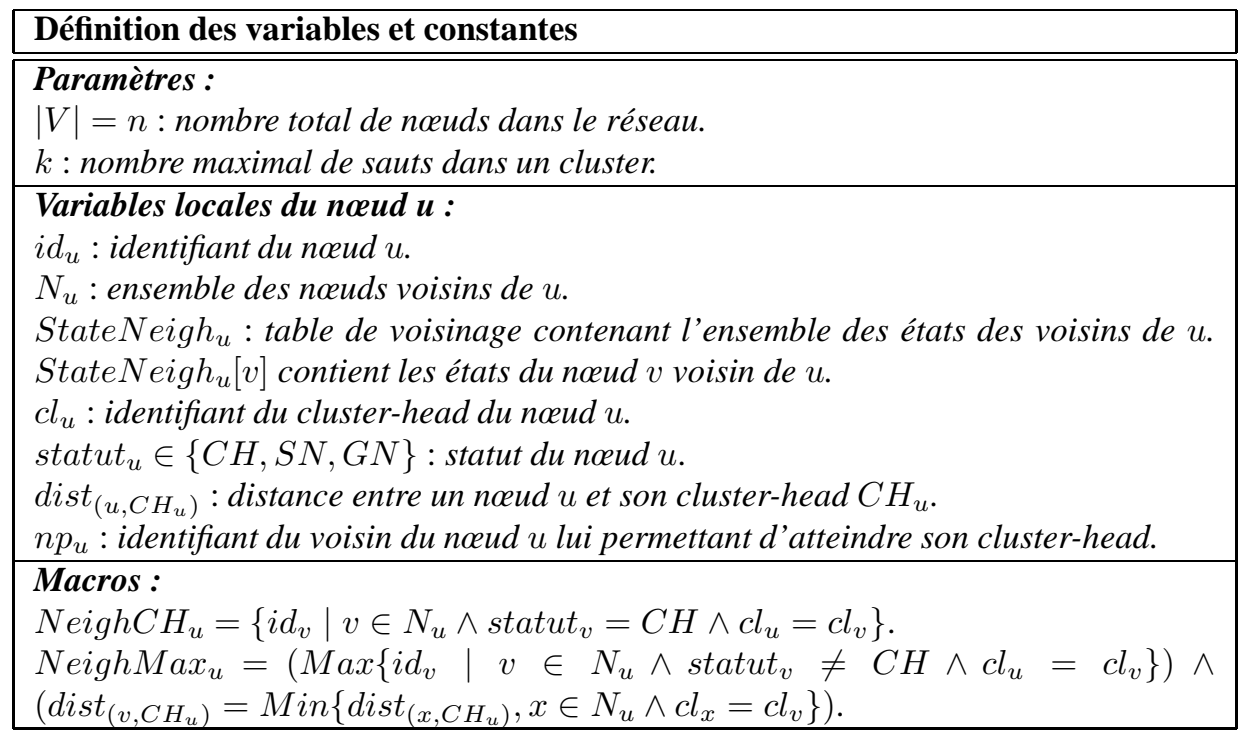

Tableau 1 - Définition des variables et constantes de notre algorithme

\subsection{Convergence et clôture}

Dans cette section, nous montrons les propriétés de convergence et de clôture de notre solution.

L'état d'un nœud est définit par la valeur de ses variables locales ainsi que celles de des ses voisins connues. Une configuration $\gamma_{i}$ du réseau est une instance de l'état de tous les nœuds. Avec notre approche, les nœuds possédant les identités les plus grandes se fixent en premier. Un nœud $u$ est dit fixé à partir de la configuration $\gamma_{i}$ si le contenu de sa variable $c l_{u}$ ne change plus. L'ensemble des nœuds fixés à $\gamma_{i}$ est noté $\mathcal{F}_{i}$. Une transition $\tau_{i}$ est le passage d'une configuration $\gamma_{i}$ à $\gamma_{i+1}$. Au cours d'une transition, chaque nœud a reçu un message d'au moins d'un voisin et a exécuté l'Algorithme 1. Ainsi, avec notre approche le nombre de nœuds fixés croit strictement à chaque configuration et tend vers $n, n$ étant le nombre de nœuds du réseau.

Lemme 6.1 Soit $\gamma_{0}$ une configuration quelconque. $A \gamma_{1}, \forall u \in V$, u est cohérent.

\section{Preuve.}

A $\gamma_{0}$ quelque soit son état, chaque nœud vérifie et corrige sa cohérence par exécution de la règle $R_{10}$ ou $R_{20}$ durant la transitions $\tau_{0}$. Ainsi, à $\gamma_{1}$ tout nœud est cohérent.

Corollaire 6.1 $\left|\mathcal{F}_{1}\right| \geq 1$.

\section{Preuve.}

Comme tous les nœuds sont cohérents d'après le lemme 6.1. Et $\exists u$ tel que $\forall v \in V$, $i d_{u}>i d_{v}$. Au moins $u$ applique $R_{11}$ durant $\tau_{0}$ et est donc fixé à $\gamma_{1}$. D'où $u \in F_{1}$ et $\left|\mathcal{F}_{1}\right| \geq 1 \Rightarrow\left|\mathcal{F}_{1}\right|>\left|\mathcal{F}_{0}\right|$. Ce nœud $u$ est noté $C H_{\text {Max }}$.

Théorème 6.1 $\forall i<k+1,\left|\mathcal{F}_{i+1}\right|>\left|\mathcal{F}_{i}\right|$ et $\mathcal{F}_{i} \subset \mathcal{F}_{i+1}$.

\section{Preuve.}


D'après le corollaire 6.1, $\left|\mathcal{F}_{1}\right|>\left|\mathcal{F}_{0}\right|$. Pour $i=0$, le résultat est vrai. A $\gamma_{2}$, nous pouvons constater que les nœuds situés à distance $1 \mathrm{du} C H_{\text {Max }}$ sont fixés soit par la règle $R_{12}$ soit par la règle $R_{16}$ selon que leur statut est $S N$ ou $C H$. Donc, $\left|\mathcal{F}_{2}\right|>\left|\mathcal{F}_{1}\right|$. Nous prouvons ensuite par récurrence qu'à $\gamma_{i}$, les nœuds situés à distance $(i-1)$ de $C H_{\text {Max }}$ se fixent par la règle $R_{13}$ ou par la règle $R_{17}$. Pour $i=k$, nous obtenons par récurrence $\left|\mathcal{F}_{k+1}\right|>\left|\mathcal{F}_{k}\right|$.

\section{Théorème 6.2 (Convergence)}

Partant d'une configuration quelconque, une configuration stable est atteinte au plus en $n+2$ transitions.

\section{Preuve.}

Comme $\left|\mathcal{F}_{1}\right| \geq 1$, nous avons $\left|\mathcal{F}_{k+1}\right|>k$. En réitérant le processus à partir d'un nouveau $C H_{\text {Max }}$ qui est le nœud d'identité maximale $\notin \mathcal{F}_{k+1}$, nous prouvons que $\forall$ $i<n,\left|\mathcal{F}_{i+1}\right|>\left|\mathcal{F}_{i}\right|$ et $\mathcal{F}_{i} \subset \mathcal{F}_{i+1}$. Pour $i=n$, nous avons $\left|\mathcal{F}_{n+1}\right|>\left|\mathcal{F}_{n}\right|$ d'où $\left|\mathcal{F}_{n+1}\right|=n$. Il faut ensuite une transition de plus pour que l'état des nœuds ne change plus. Nous obtenons un temps de stabilisation d'au plus $n+2$ transitions.

\section{Théorème 6.3 (Clôture)}

A partir d'une configuration légale $\gamma_{i}$, sans occurrence de fautes, chaque noud restera dans une configuration légale.

Preuve. Soit $\gamma_{i}$ une configuration légale, $\forall u \in V u$ est fixé et seule la règle $R_{0}$ s'exécutera. Nous aurons donc $\forall j>i$, à $\gamma_{j}$ une configuration légale.

Remarque 6.1 (Pire des cas)

Avec notre approche, nous observons le pire des cas dans une topologie où les nouds forment une chaîne ordonnée. Dans une telle topologie, comme illustré au niveau de la figure 5, les nouds se fixent du plus grand au plus petit et le temps de stabilisation est de $n+2$ transitions.

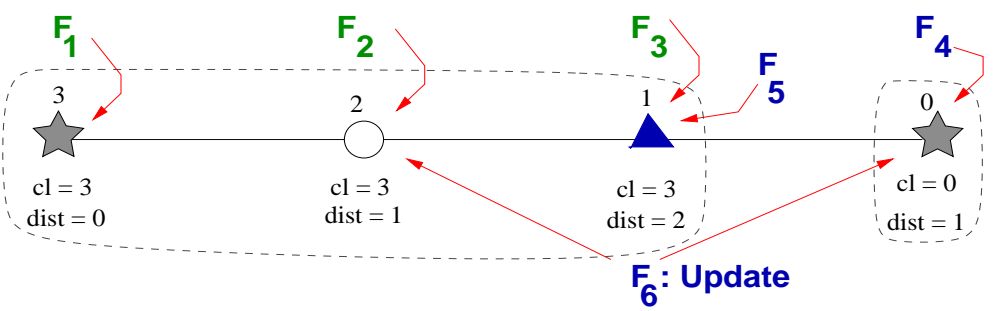

Figure 5 - Stabilisation dans une chaîne ordonnée

\subsection{Occupation mémoire}

Dans cette section, nous montrons l'occupation mémoire maximale nécessaire dans dans notre solution.

Lemme 6.2 L'espace mémoire nécessaire pour chaque voisin est $\log (2 n+3+k)$ bits.

\section{Preuve.}

Pour fonctionner, chaque nœud $u$ du réseau a besoin de connaître pour chacun de ses voisins $v \in N_{u}$ son l'identifiant $\left(i d_{v}\right)$, son identifiant de cluster $\left(c_{v}\right)$, son statut statut $_{v}$ ) et sa distance $\operatorname{dist}_{\left(v, C H_{v}\right)}$. Ainsi, pour un réseau de $n$ nœuds, nous avons au plus : 
- $n$ valeurs possibles pour l'identifiant du nœud;

- $n$ valeurs possibles pour l'identifiant de cluster;

- trois (3) valeurs de statut différentes que sont $C H, S N$ et $G N$;

- $k$ valeurs possibles pour la distance.

Or, il est nécessaire d'avoir $\log (n)$ bits pour encoder une variable avec $n$ valeurs possibles. Donc, pour chaque nœud voisin, l'algorithme a besoin d' au plus de $\log (2 n+k+3)$ bits.

Corollaire 6.2 Chaque næud $u$ a besoin de $\left(\Delta_{u}+1\right) * \log (2 n+3+k)$ bits.

\section{Preuve.}

D'après le lemme 6.2, chaque voisin $v$ d'un nœud $u$ requiert $\log (2 n+k+3)$ bits. Soit $N_{u}$ le voisinage du nœud $u$. Posons $\Delta_{u}=\left|N_{u}\right|$ comme étant le degré - nombre de voisins - du nœud $u$. Or, pour l'exécution de l'algorithme, chaque nœud $u$ a besoin de connaître l'état de touts ses $\Delta_{u}$ voisins. De plus, $u$ mémorise aussi les mêmes informations pour lui.

Donc, chaque nœud $u$ a besoin de $\left(\Delta_{u}+1\right) * \log (2 n+3+k)$ bits.

Corollaire 6.3 Chaque nøud u a besoin d'au plus $n * \log (2 n+3+k)$ bits.

\section{Preuve.}

Pour un réseau de $n$ nœuds, le voisinage $N_{u}$ de chaque nœud $u$ peut contenir au maximum $n-1$ voisins (pour un graphe complet). Dans ce cas précis, pour fonctionner, notre algorithme a besoin de $n * \log (2 n+3+k)$ bits par nœud.

\subsection{Discussions}

Nous venons de montrer avec le corollaire 6.3 que nous observons l'occupation mémoire la plus importante dans le cas d'un graphe complet où chaque nœud a besoin de $n * \log (2 n+3+k)$ bits. Or, comme dans un graphe complet tous les nœuds sont voisins (c.f. Figure 6(a)), nous pouvons aisément remarquer qu'en 2 transitions tous les nœuds se fixent. En effet, dans un graphe complet, partant d'une configuration quelconque $\gamma_{0}$, à la configuration $\gamma_{1}$, le nœud possédant la plus grande identité se fixe en premier. Puis, à la configuration $\gamma_{2}$, tous les autres nœuds se fixent au nœud d'identité maximale. Et nous obtenons un seul cluster. Dans ce cas, l'occupation mémoire est maximale et le temps de stabilisation est minimal.

Cependant, comme nous l'avons montré dans la section 6.1, pour l'autre critère déterminant de algorithme, à savoir le temps de stabilisation, nous observons le pire des cas dans une topologie en chaîne ordonnée. Or, comme illustré dans l'exemple de la chaîne ordonnée de la figure 6(b), les nœuds situés au deux extrémités dans la chaîne ont un (1) seul voisin alors que ceux à l'intérieur de la chaîne n'ont que deux (2) voisins. Dans ce cas, l'occupation mémoire est minimale et le temps de stabilisation est maximale.

En résumé, dans le cas où nous observons un temps de stabilisation au maximal (chaîne ordonnée), l'occupation mémoire est la moindre. Et inversement, dans le cas ou l'occupation mémoire est au maximale (graphe complet), le temps de stabilisation est au plus petit. 


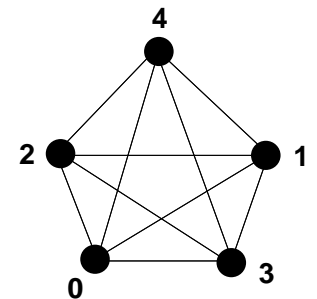

(a) Graphe complet

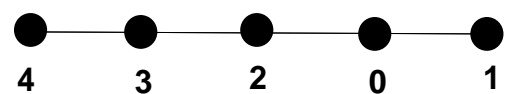

(b) Chaîne ordonnée

Figure 6 - Graphe complet vs Chaîne Ordonnée

\subsection{Comparaison analytique}

Le tableau 2, illustre une comparaison du temps de stabilisation, de l'espace mémoire par voisin et du voisinage de notre algorithme avec ceux des meilleurs solutions de clustering auto-stabilisantes à $k$ sauts fondées soit sur un sur un modèle à états $[16,13]$ soit sur un modèle synchrone à passage de messages [26].

Dans un premier temps, nous pouvons noter que le temps de stabilisation de notre solution n'est pas fonction du paramètre $k$ contrairement aux solutions proposées par dans $[13,26]$. Étant donné que notre approche combine la découverte de voisinage et la procédure de clustering en une seule phase, elle présente un unique temps de stabilisation contrairement à la solution de Datta et al. [16] et de Larsson and Tsigas [26]. Dans ces deux solutions, les auteurs commencent d'abord par construire l'ensemble de clusterheads. Puis, partant de cet ensemble, ils structurent le réseaux en clusters de rayon $k$.

Nous pouvons aussi remarquer que notre solution considère un voisinage à distance 1 pour construire des clusters à $k$ sauts. Alors que dans [16] et [13], les processus ont respectivement des rayons de lecture de $k$ et $k+1$ sauts. Cependant, nous notons que notre algorithme nécessite plus de mémoire par nœud comparé aux solutions qui utilisent un modèle à états [16, 13]. En effet, contrairement aux solutions décrites dans [16] et [13], nous utilisons un modèle asynchrone à passage de messages. Or, dans un tel modèle, chaque nœud mémorise les informations définissant les états de ses voisins. Ce qui n'est pas le cas dans un modèle à états qui est utilisé dans [16, 13].

Remarquons néanmoins que notre algorithme requiert moins de mémoire que la solution de Larsson et Tsigas [26] qui utilise un modèle synchrone à passage de messages. En effet, dans la solution de Larsson et Tsigas [26], les messages sont retransmis jusqu'à une distance $k$ alors que dans la notre, les échanges de messages se font uniquement entre voisins de distance 1 .

\begin{tabular}{|c|c|c|c|}
\hline & Temps de stabilisation & Occupation mémoire par nœud & Voisinage \\
\hline Notre solution & $n+2$ & $\log (2 n+k+3)$ & 1 saut \\
\hline Caron et al. $[13]$ & $O(n * k)$ & $O(\log (n)+\log (k))$ & k+1 sauts \\
\hline Datta et al. $[16]$ & $O(n), O\left(n^{2}\right)$ & $O(\log (n))$ & k sauts \\
\hline Larsson et Tsigas [26] & $O(k), O(g * k * \log (n))$ & $O\left(\left|G_{i}^{k}\right| *(\log (n)+\log (k))\right)$ & k saut \\
\hline
\end{tabular}

Tableau 2 - Comparaison analytique du temps de stabilisation, de l'occupation mémoire et du voisinage 


\section{7. Évaluation de performances moyennes}

Dans cette section, nous présentons une implémentation de notre algorithme et une évaluation de ses coûts et performances moyennes par simulation.

Comme nous l'avons montré dans la Section 6, notre réseau converge dans le pire des cas en $n+2$ transitions. Ceci correspond au cas le plus défavorable d'une topologie où les nœuds forment une chaine ordonnée. Or, un réseau $A d$ Hoc est caractérisé par une topologie dense et aléatoire. Ainsi, nous faisons recours à une campagne de simulation pour évaluer les performances moyennes de notre algorithme.

Nous avons utilisé le simulateur $O M N e T++$ [31] et la librairie SNAP [1] comme générateur de graphes aléatoires. Toutes nos simulations sont effectuées sous Grid'5000 [12]. De plus, nous fixons un intervalle de confiance de $99 \%$ et nous fournissons des valeurs moyennes sur une série de 100 simulations pour chaque taille de réseaux.

\subsection{Impact de la taille du réseaux et du degré des nœuds}

Nous entamons l'évaluation des performances moyennes de notre solution en étudiant l'impact de la densité et du nombre de nœuds du réseau sur le temps de stabilisation du réseau.
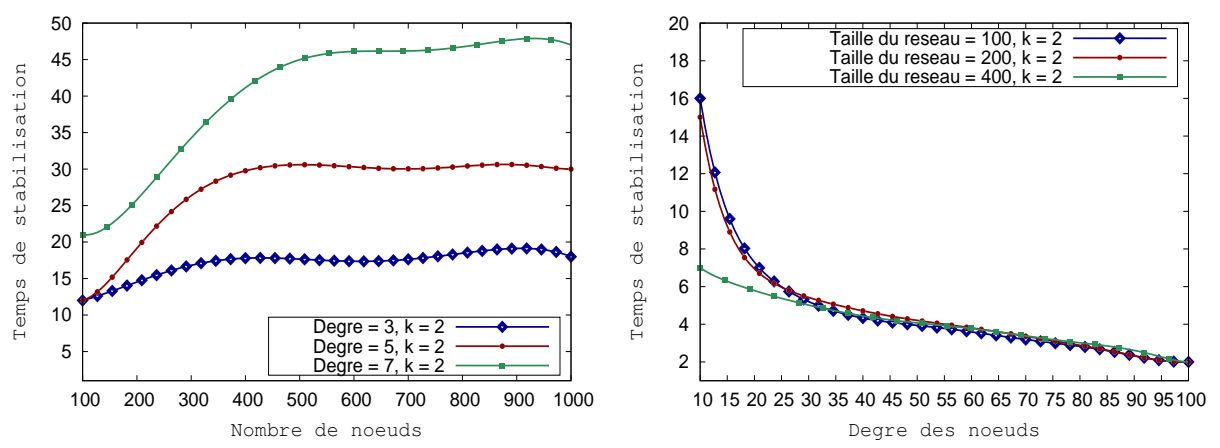

(a) Temps de stabilisation en fonction du nombre de (b) Temps de stabilisation en fonction du degré des nœuds nœuds

Figure 7 - Impact de la taille du réseaux et du degré des nœuds sur le temps de stabilisation

Dans la première série d'expériences présentée au niveau la figure 7(a), nous fixons le paramètre $k=2$ et nous faisons varier le nombre de nœuds de 100 à 1000 par pas de 100. Pour chaque taille de réseau fixée, à l'aide de la librairie SNAP, nous générons des graphes aléatoires $\delta$-réguliers, où $\delta$ est reprendre degré des nœuds (nombre de voisins). Nous fixons arbitrairement le degré $\delta$ à 3,5 et 7 . Puis, pour chaque taille de réseau, nous calculons le temps de stabilisation comme étant le nombre maximal de transitions obtenus jusqu'à la formation des clusters stables.

Ainsi, au niveau de la figure 7(a), nous remarquons que le temps de stabilisation moyen augmente légèrement avec l'augmentation du nombre de nœuds mais varie peu à partir d'une certaine taille du réseau. De plus, nous notons que pour des topologies totalement aléatoires, le temps de stabilisation moyen est très en dessous de $n+2$ transitions (valeur formelle prouvée dans le pire des cas). 
Pour mieux observer l'impact du degré des nœuds sur le temps de stabilisation, comme illustré avec la figure 7(b), nous fixons la taille du réseau et nous faisons varier le degré de nœuds.

Nous observons que pour des tailles de réseau fixées à 100, 200 et 400 nœdus (cf. Figure 7(b)), plus le degré des nœuds augmente, plus le temps de stabilisation diminue. En effet, si le degré augmente, les nœuds ayant les plus grandes identités du réseau ont plus de voisins. Donc, ils attirent plus de nœuds dans leurs clusters durant chaque transition. Ainsi, avec notre approche, plus le degré des nœuds augment, plus le temps de stabilisation diminue. Or, les réseaux Ad Hoc sont souvent caractérisés par une forte densité.

\section{2. Étude du passage à l'échelle et impact du paramétrer $k$}

Pour étudier le passage à l'échelle de notre solution, nous faisons varier le nombre de nœuds dans le réseau en même temps que la densité de connexité du réseau. Pour $k=2$, nous faisons évoluer le nombre de nœuds de 100 à 1000 par pas de 100. Pour chaque taille de réseau fixée, nous faisons varier la densité du réseau de $10 \%$ à $100 \%$ par pas de 10 en générant des graphes aléatoires suivant le modèle de Erdos Renyi [18] à l'aide de la librairie SNAP. Nous obtenons la courbe $3 D$ de la figure $8(a)$.

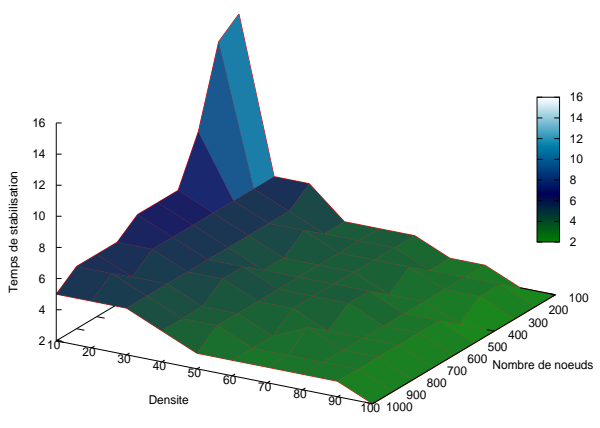

(a) Passage à l'échelle

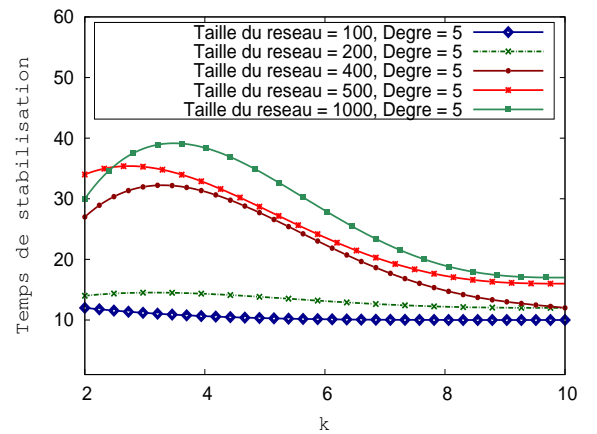

(b) Impacte du paramètre $k$

Figure 8 - Passage à l'échelle - impacte du paramètre $k$

Nous remarquons que sauf pour de faibles densités (10\% et $20 \%$ ), le temps de stabilisation varie légèrement avec l'augmentation du nombre de nœuds. Et cas de faibles densités, nous observons un pic. Mais avec l'augmentation du nombre de nœuds, le temps de stabilisation diminue et nous observons le même phénomène que la figure 7(b). Avec cette série de simulations, nous pouvons soulever deux observations. (i) Seule la densité de connexité est le facteur déterminant avec notre approche. (ii) En moyenne, pour des réseaux avec une topologie quelconque, le temps de stabilisation est très inférieur à celui du pire des cas ( $n+2$ transitions). Rappelons que ce pire cas est une topologie où les nœuds forment une chaîne ordonnée comme nous l'avons prouvé dans Section 6.

Pour analyser l'impact du paramètre $k$, nous fixons arbitrairement un degré de 5 et nous considérons des réseaux de taille 100, 200, 400, 500 et 1000 nœuds. Pour chaque taille de réseau, nous faisons varier la valeur de $k$ de 2 à 10. La figure 8(b) montre le temps de stabilisation en fonction de la valeur de $k$. Nous observons une diminution du temps de stabilisation avec l'augmentation de la valeur de $k$. En effet, comme les messages hello échangés contiennent la valeur de la distance $k$, si $k$ augmente, le champs d'influence des 
nœuds avec une plus grande identité augmente. Les nœuds effectuent moins de transitions pour se fixer à un $C H$. Avec de petites valeurs du paramètre $k$, nous avons des clusters de faibles diamètres. Donc, il nécessite plus de transitions pour atteindre un état stable dans tous les clusters. Notons que quelle que soit la valeur du paramètre $k$ et pour des graphes de réseaux totalement aléatoires, nous obtenons des temps de stabilisation très inférieurs au pire des cas $(n+2$ transitions).

\section{3. Évaluation du nombre de clusters}

Avec la campagne de simulation présentée au niveau de la figure 9, nous évaluations nombre clusters construit par notre solution. Pour ce faire, nous calculons le pourcentage de clusters construit comme étant le nombre cluster-head obtenu sur le nombre total de nœuds dans le réseau. Cette évaluation s'est faite en fonction du degré moyen du réseau et en fonction du paramètre $k$.
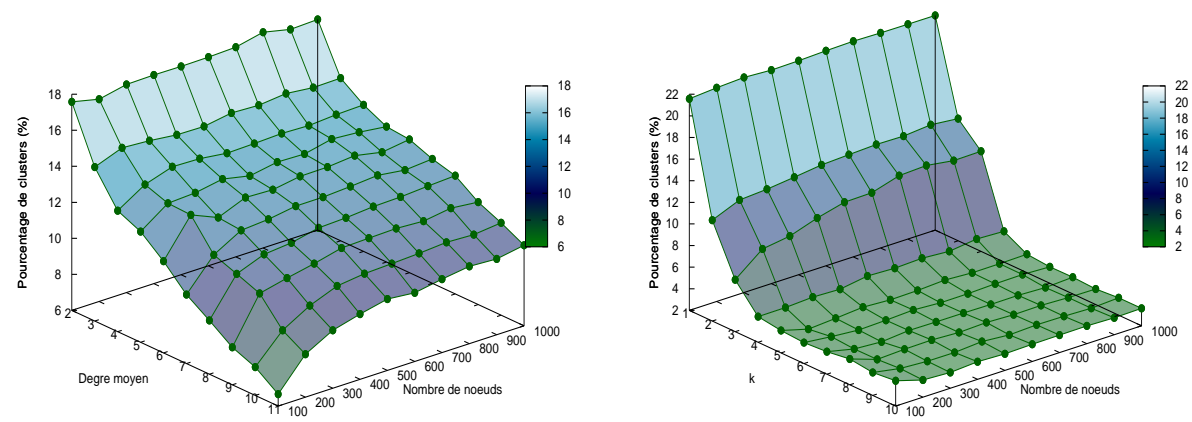

(a) Pourcentage de clusters construit en fonction du de- (b) Pourcentage de clusters construit en fonction du pagré du réseau ramètre $k$

Figure 9 - Pourcentage de clusters

Dans la figure 9(a), nous fixons le paramètre $k=2$ puis, nous faisons varier le nombre de nœuds de 100 à 1000 . Pour chaque taille de réseau fixée, nous faisons varier le degré moyen de 2 à 11. Nous observons d'abord que, pour chaque valeur du degré moyen fixée, le pourcentage de clusters varie peu avec la l'augmentation de la taille du réseau. Ceci atteste qu'en termes de pourcentage de clusters construit, notre solution assure le passage à l'échelle. Par ailleurs, nous remarquons que, pour chaque taille de réseau fixée, le pourcentage de clusters construit diminue avec l'augmentation du degré moyen dans le réseau. En effet, l'augmentation du degré moyen entraîne un plus grand nombre de voisins pour les nœuds d'identité plus grande. Ainsi, ces derniers attirent dans leurs clusters un nombre plus important de nœuds. D'où une diminution du pourcentage de clusters

Au niveau de la figure 9(b), nous fixons un degré moyen de 6 voisins puis, nous faisons varier le nombre de nœuds de 100 à 1000 . Pour chaque taille de réseau fixée, nous faisons évoluer le paramètre $k$ de 1 à 10 . Nous observons d'abord que pour chaque valeur du paramètre $k$ fixée, le taux de cluster-head varie très peu avec l'augmentation du nombre de nœuds dans le réseau. Ceci atteste du passage à l'échelle de notre solution pour le pourcentage de clusters construit en fonction fonction du paramètre $k$. Par ailleurs, nous notons une forte chute du taux de cluster-head avec suivant l'augmentation du paramètre 
$k$. En effet, avec l'augmentation du paramètre $k$, le diamètre des clusters augmente. Ainsi, nous obtenons des clusters contenant plus de nœuds. D'où une diminution du pourcentage de cluster-head. Notons aussi que les valeurs du paramètre qui donnent les pourcentages de cluster-head les plus intéressant sont 2 et 3 . Au delà de 3 , nous commençons à obtenir des clusters de diamètres plus grands.

La figure 9(a) nous montre que, selon le degré du réseau, notre approche construit un taux de cluster-head entre $6 \%$ et $18 \%$. La figure 9 (b) quant à elle illustre que, pour les valeurs les plus intéressantes du paramètre $k$ (2 et 3), nous obtenons un taux de clusterhead entre $7 \%$ et $16 \%$. Plusieurs études, comme [2, 14, 32], ont montré que le nombre optimal de cluser-heads doit correspondre entre $5 \%$ et $20 \%$ du nombre total de nœuds dans le réseau.

\subsection{Tolérance aux fautes transitoires : évaluation du coût de re-clustering}

Dans les réseaux Ad Hoc, la mobilité des nœuds entrâne des changements de topologie qui peuvent occasionner des fautes transitoires par modification de la structure des clusters. Ceci peut arriver lorsqu'un nœud jouant le rôle de cluster-head venait à changer de position dans le réseau. De plus, un nœud peut disparaître du réseau du fait l'épuisement de son énergie, d'une panne de son module de communication ou d'autres facteurs externes dûs à l'environnement de déploiement qui est souvent hostile.

Nous supposons la mobilité (arrivée ou départ d'un nœud dans un cluster) ou la disparition d'un nœud entrainant un changement dans le cluster comme une faute transitoire. Celle-ci est alors détectée et corrigée automatiquement par l'algorithme 1 sans intervention extérieure. Nous considérons qu'une faute transitoire survient après la stabilisation c'est à dire après la formation de clusters stables. En effet, une faute lors de la phase de clustering est transparente. La détection d'une faute s'effectue toujours à l'aide des messages hello. Un nœud qui ne reçoit plus de réponses d'un voisin après un temps bien déterminé le considère comme disparu ou ayant changé de position dans le réseau. Ainsi, nous évaluons l'impact de fautes transitoires en mesurant le coût de la procédure de re-clustering en termes de nombre de transitions supplémentaires pour retrouver un état stable et le nombre de nœuds impactés.

Dans la série d'expériences illustrée au niveau de la figure 10, nous fixons un réseau de 1000 nœuds avec un degré moyen de 6 . Ensuite, nous exécutons l'algorithme 1 jusqu'à la stabilisation. Puis, nous introduisons des fautes transitoires en faisant disparaître aléatoirement 1 à $5 \%$ des nouds du réseau. Pour chaque pourcentage de nœuds disparus, une série de 100 simulations est effectuée.

Dans la figure 10(a), nous calculons le temps de stabilisation maximal comme étant le nombre de transitions supplémentaires nécessaire pour retrouver un nouvel état légal. Nous observons que le nombre de transitions nécessaire pour corriger les fautes transitoires augmente en suivant l'augmentation du taux de nœuds disparus. En effet, comme illustré dans la figure 10(b), l'augmentation du pourcentage de nouds disparus fait crôtre logiquement le nombre nœuds impactés dans le réseau. De ce fait, il nécessite plus de transitions pour que le réseau devient stable à nouveau. Cependant, une disparation jusqu'à $5 \%$ des nœuds du réseau n'a d'impact que sur environs $1 / 4$ des nœuds du réseau. Ceci atteste que les fautes transitoires n'impactent que les clusters dans lesquels elles se sont produites et éventuellement les clusters adjacents. Également, nous observons que 
pour une disparition jusqu'à $5 \%$, le nombre de transitions supplémentaires requis pour que le réseau retrouve un état stable est très moindre comparé au temps de stabilisation en partant d'une configuration quelconque (cf. Figure 7 et Figure 8(a)).

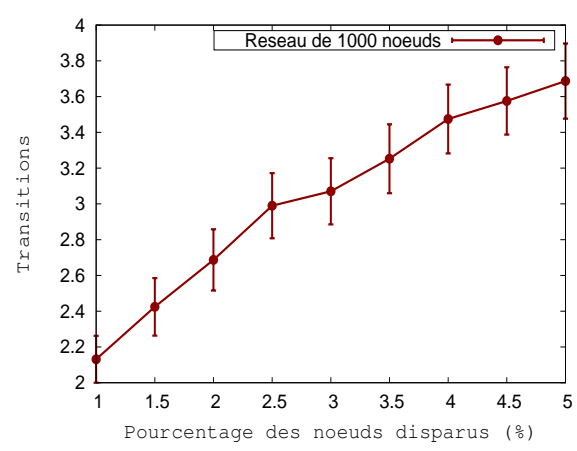

(a) Transitions supplémentaires

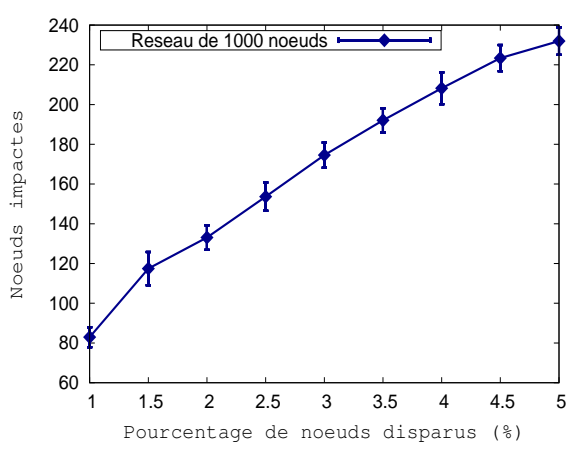

(b) Pourcentage de nœuds impactés

Figure 10 - Impact de la disparation de 1 à $5 \%$ de nœuds

Les résultats expérimentaux illustrés dans la figure 10 montrent que la correction de fautes transitoires nécessite un nombre moindre de transitions. En effet, les fautes transitoires impactent les cluters dans lesquels elles se sont produites et éventuellement les clusters adjacents. De plus, les nœuds réutilisent les informations déjà présentes dans leurs tables de voisinage pour la correction de fautes transitoires. Ainsi, notre solution s'adapte aux fautes transitoires dues à des modifications topologiques.

\subsection{Discussions}

Dans cette section, nous venons d'évaluer les performances moyennes de notre solution. Les résultats de simulation ont notamment montré que pour des topologies réseaux suivant des graphes totalement aléatoires, nous obtenons des temps de stabilisation très inférieurs à celui du pire des cas. Rappelons que, comme montré dans la Section 6 , le pire des cas correspond à la topologie particulière de chaîne ordonnée. Dans ce cas, nous obtenons un temps de stabilisation de $n+2$ transitions. De plus, en cas fautes transitoires, les simulations montrent d'une part que le nombre de transitons nécessaire pour retrouver un état stable est moindre. D'autre part, les fautes transitoires impactent les cluters dans lesquels elles se sont produites et éventuellement les clusters adjacents.

Dans [7, 8], nous avons appliqué notre algorithme dans le cadre des Réseaux de Capteurs Sans Fil (RCSF) avec contrainte énergétique. Nous avons montré que notre solution est générique et complète. En plus du critère principal d'élection du cluster-head (identité maximale), notre algorithme peut s'utiliser aisément avec d'autres critères tels que le degré des nœuds ou l'énergie résiduelle. Nous avons validé cette étude en évaluant, suivant plusieurs critère d'élection du cluster-head, le coût de communication en termes de nombre total de messages échangés jusqu'à la formation des clusters stables dans tout le réseau et la consommation énergétique qui en découle. Les études que nous avons mené dan $[7,8]$ montrent que le critère d'identité maximale apporte plus de stabilité lors de la construction des clusters. Rappelons que pour mesurer la consommation énergétique, nous avons implémenté le modèle énergétique de référence dans les RCSF proposé par Heinzelman et al. [20]. 
Également, dans [6], nous avons mené une étude comparative, toujours dans le contexte des RCSF avec contrainte énergétique, de notre solution avec l'approche proposée par Mitton et $a l$. dans [27]. En effet, à notre connaissance, l'algorithme de Mitton et al. est la solution de référence dans notre modèle. C'est à dire un algorithme de clustering autostabilisant à $k$ sauts, distribué et utilisant un modèle asynchrone à passage de messages. Nous avons comparé les deux approches, sous le même modèle et environnement de tests, en évaluant le coût de communication et la consommation énergétique. Les résultats obtenus dans [6] montrent que notre approche réduit le coût de communication et la consommation énergétique par un facteur 2 comparé à la solution de Mitton et al. [27] .

\section{Conclusion}

Dans cet article, nous avons présenté un algorithme complètement distribué et autostabilisant pour structurer le réseau en clusters non-recouvrants de diamètre au plus $2 k$. Sans initialisation, notre solution n'utilise que des informations provenant des nœuds voisins à distance 1. Elle combine la découverte du voisinage et la construction des clusters.

Nous avons montré que, partant d'un état quelconque, un état stable est atteint en au plus $n+2$ transitions. De plus, il nécessite une occupation mémoire de $\Delta_{u} * \log (2 n+k+3)$ bits pour chaque nœud $u$ où $\Delta_{u}$ représente le degré - nombre de voisins - de $u, n$ le nombre total de nœuds et $k$ la distance maximale dans les clusters.

Les compagnes de simulation, menées sous OMNeT++, montrent d'une part que pour des réseaux totalement aléatoires, le temps de stabilisation moyen est très inférieur à celui du pire des cas. D'autre part, après l'occurrence de fautes transitoires, le nombre de transitions supplémentaires pour retrouver un nouvel état stable et le nombre de nœuds impactés sont moindres.

Actuellement, nous travaillons sur l'élaboration d'un protocole de routage avec aggravation de donnée utilisant la structure de nos clusters dans le but d'optimiser l'acheminement de l'information.

\section{Remerciements}

Ces travaux ont été effectués dans le cadre du projet CPER CapSec ROFICA cofinancé par la région de Champagne-Ardenne et le FEDER. Nous remercions également le Centre de Calcul de Champagne-Ardenne ROMEO ${ }^{1}$ et Grid' 5000.

\section{Bibliographie}

[1] 2013. SNAP : Stanford Network Analysis Platform.

1. Le Centre de Calcul de Champagne-Ardenne ROMEO est une plateforme technologique de l'Université de Reims Champagne-Ardenne soutenue par la région Champagne-Ardenne depuis 2002. Pour plus d'information : https ://romeo.univ-reims.fr 
[2] Navid Amini, Alireza Vahdatpour, Wenyao Xu, Mario Gerla, and Majid Sarrafzadeh. Cluster size optimization in sensor networks with decentralized cluster-based protocols. Computer Communications, pages $207-220,2012$.

[3] A.D. Amis, R. Prakash, T.H.P. Vuong, and D.T. Huynh. Max-min d-cluster formation in wireless ad hoc networks. In INFOCOM 2000, pages 32-41, 2000.

[4] H. Attiya and J. Welch. Distributed computing : fundamentals, simulations, and advanced topics. Wiley series on Parallel and Distributed Computing. 2004.

[5] Mandicou Ba, Olivier Flauzac, Bachar Salim Haggar, Florent Nolot, and Ibrahima Niang. SelfStabilizing k-hops Clustering Algorithm for Wireless Ad Hoc Networks. In 7th ACM IMCOM (ICUIMC), pages $38: 1-38: 10,2013$.

[6] Mandicou Ba, Olivier Flauzac, Rafik Makhloufi, Florent Nolot, and Ibrahima Niang. Comparison Between Self-Stabilizing Clustering Algorithms in Message-Passing Model. In 9th ICAS, pages 27-32, 2013.

[7] Mandicou Ba, Olivier Flauzac, Rafik Makhloufi, Florent Nolot, and Ibrahima Niang. EnergyAware Self-Stabilizing Distributed Clustering Protocol for Ad Hoc Networks : the case of WSNs. KSII Transactions on Internet and Information Systems, page 19p, 2013. To appear.

[8] Mandicou Ba, Olivier Flauzac, Rafik Makhloufi, Florent Nolot, and Ibrahima Niang. Evaluation Study of Self-Stabilizing Cluster-Head Election Criteria in WSNs. In 6th CTRQ, pages 64-69, 2013.

[9] Lalia Blin, Maria Gradinariu Potop-Butucaru, and Stephane Rovedakis. Self-stabilizing minimum degree spanning tree within one from the optimal degree. JPDC, pages $438-449$, 2011.

[10] Alain Bui, Abdurusul Kudireti, and Devan Sohier. A fully distributed clustering algorithm based on random walks. IPDPS, pages 125-128, 2009.

[11] Alain Bui, Devan Sohier, and Abdurusul Kudireti. A random walk based clustering with local recomputations for mobile ad hoc networks. IPDPSW, pages 1-8, 2010.

[12] F. Cappello and et al. Grid'5000 : A large scale and highly reconfigurable grid experimental testbed. In GRID, pages 99-106, 2005.

[13] Eddy Caron, Ajoy K. Datta, Benjamin Depardon, and Lawrence L. Larmore. A self-stabilizing k-clustering algorithm for weighted graphs. JPDC., pages 1159-1173, 2010.

[14] Ali Chamam and Samuel Pierre. A distributed energy-efficient clustering protocol for wireless sensor networks. Computers \& Electrical Engineering, pages 303 - 312, 2010.

[15] Ajoy Datta, Lawrence Larmore, and Priyanka Vemula. Self-stabilizing leader election in optimal space. In SSS, pages 109-123. 2008.

[16] Ajoy K. Datta, Stéphane Devismes, and Lawrence L. Larmore. A self-stabilizing O(n)-round k-clustering algorithm. In SRDS, pages 147-155, 2009.

[17] Edsger W. Dijkstra. Self-stabilizing systems in spite of distributed control. Communications of the ACM, pages 643-644, 1974.

[18] P. Erdos and A. Renyi. On the evolution of random graphs. Publication of the Mathematical Institute of the Hungarian Academy of Sciences, pages 17-61, 1960.

[19] Olivier Flauzac, Bachar Salim Haggar, and Florent Nolot. Self-stabilizing clustering algorithm for ad hoc networks. ICWMC, pages 24-29, 2009.

[20] Wendi Rabiner Heinzelman, Anantha Chandrakasan, and Hari Balakrishnan. Energy-efficient communication protocol for wireless microsensor networks. In HICSS, 2000.

[21] Colette Johnen and Fouzi Mekhaldi. Self-stabilization versus robust self-stabilization for clustering in ad-hoc network. In 17th Euro-Par, pages 117-129, 2011.

[22] Colette Johnen and Le Nguyen. Self-stabilizing weight-based clustering algorithm for ad hoc sensor networks. In ALGOSENSORS, pages 83-94. 2006. 
140 A R I M A - Volume $17-2014$

[23] Colette Johnen and Le Huy Nguyen. Robust self-stabilizing weight-based clustering algorithm. TCS, pages $581-594,2009$.

[24] Colette Johnen and LeHuy Nguyen. Self-stabilizing construction of bounded size clusters. ISPA, pages 43-50, 2008.

[25] J. Kuroiwa, Y. Yamauchi, Weihua Sun, and M. Ito. A self-stabilizing algorithm for stable clustering in mobile ad-hoc networks. In 4th IFIP NTMS, pages 1-7, 2011.

[26] Andreas Larsson and Philippas Tsigas. Self-stabilizing (k,r)-clustering in wireless ad-hoc networks with multiple paths. In 14th OPODIS, pages 79-82, 2010.

[27] N. Mitton, E. Fleury, I. Guerin Lassous, and S. Tixeuil. Self-stabilization in self-organized multihop wireless networks. In ICDCSW, pages 909-915, 2005.

[28] Nathalie Mitton, Anthony Busson, and Eric Fleury. Self-organization in large scale ad hoc networks. In MED-HOC-NET, 2004.

[29] Gregory J Pottie and William J Kaiser. Wireless integrated network sensors. Communications of the ACM, pages 51-58, 2000.

[30] G. Tel. Introduction to Distributed Algorithms. Cambridge University Press, 2000.

[31] András Varga and Rudolf Hornig. An overview of the OMNeT++ simulation environment. In Simutools, pages $60: 1-60: 10,2008$.

[32] Ossama Younis and Sonia Fahmy. Heed : a hybrid, energy-efficient, distributed clustering approach for ad hoc sensor networks. IEEE Transactions on Mobile Computing, pages 366 379,2004

[33] Jiguo Yu, Yingying Qi, Guanghui Wang, and Xin Gu. A cluster-based routing protocol for wireless sensor networks with nonuniform node distribution. AEU - International Journal of Electronics and Communications, pages 54-61, 2012. 